\title{
Do Equol's C-ring Hydrogens Contribute to Free Radical Scavenging?
}

\author{
Ana Amić1 ${ }^{*}$, Dejan Milenkovićc,4, Jasmina Dimitrić Marković3, Zoran Markovićc \\ ${ }^{1}$ Department of Chemistry, Josip Juraj Strossmayer University of Osijek, 8/A Ulica cara \\ Hadrijana, 31000 Osijek, Croatia \\ e-mail: aamic@kemija.unios.hr \\ ${ }^{2}$ Bioengineering Research and Development Center, 6 Prvoslava Stojanovića Street, 34000 \\ Kragujevac, Serbia \\ e-mail: deki82@kg.ac.rs \\ 3 The Faculty of Physical Chemistry, University of Belgrade, Studentski trg 12-16, 11000 \\ Belgrade, Serbia \\ e-mail: markovich@ffh.bg.ac.rs \\ ${ }^{4}$ Institute of Information Technologies, Department of Science, University of Kragujevac, \\ Jovana Cvijica bb, 34000 Kragujevac, Serbia \\ e-mail: zmarkovic@uni.kg.ac.rs \\ *corresponding author
}

\begin{abstract}
Free radical scavenging potency of physiologically active equol molecule, derived by gut microbiota from soy isoflavone daidzein, was investigated by using M06-2X/6-311++G(d,p) level of theory, accompanied with the TST and Eckart tunneling corrections for the estimation of rate constants. Phenolic hydrogens of equol are recognized as much more abstractable than $\mathrm{C}$-ring hydrogens. This finding is opposite to the one very recently suggested, but in accordance with well-known facts related to the flavonoid chemistry and phenolic $\mathrm{O}-\mathrm{H}$ vs $\mathrm{C}-\mathrm{H}$ reactivity.
\end{abstract}

Keywords: Equol, free radical scavenging, fHAT mechanism, tunneling

\section{Introduction}

Epidemiological studies suggest that diets rich in soy isoflavones are beneficial to human health (Mayo et al. 2019). The perceived health promoting effects of such eating habits are ascribed not to parental forms of ingested isoflavones but mainly to their intestinal metabolites ( $\mathrm{Li}$ 2019). Among metabolites, equol has been extensively studied because of its antioxidant (Rüfer and Kulling 2006) and estrogenic activity (Setchell et al. 2005) which may reduce the incidence of age-related and estrogen-dependent disorders (Mayo et al. 2019).

Isoflavone equol is not naturally present in most fruits and vegetables. Until now, it has only been found in white cabbage (Hounsome et al. 2009). Equol is primarily a product of human and animal intestinal bacterial metabolism of dietary soy isoflavone daidzein (Jackson et al. 2011). Due to a lack of active intestinal bacterial flora, less than half of human population (mainly Asians) is able to produce equol after consumption of soy foods (Watanabe and Uehara 
2019). Equol in cow's milk is derived from forage legumes such as red clover (Mustonen et al. 2009).

Free radicals play important role in normal physiological cell functions. However, elevated levels of free radicals that overcome endogenous antioxidant enzymes capacity cause a condition known as oxidative stress and damage cell biomolecules. Many diseases arise from overproduction of free radicals (Finkel and Holbrook 2000). In order to restore homeostasis, consumption of food rich in antioxidants may help. It has been shown that individuals with soyrich diets, in which isoflavones are particularly abundant, have significantly lower occurrences of cardiovascular disease, osteoporosis, and some types of cancer (Yuan et al. 2007).

In vivo equol may act as an antioxidant by stimulating antioxidant enzymes (catalase, glutathione peroxidase and superoxide dismutase) activity and expression to combat oxidative stress condition (Choi 2009). Equol is superior to other isoflavones (considered as weak antioxidants when tested in vitro) in inhibition of LDL oxidation and membrane lipid peroxidation (Wiseman et al. 2000), as well as in different antioxidant and free radical scavenging assays (Mitchell et al. 1998, Rimbach et al. 2003). Among possible mechanisms of protective action of isoflavones, direct scavenging of free radicals may be operative (Galleano et al. 2010). It could be of physiological relevance in situ, i.e., in gastrointestinal tract where metabolites such as equol may reach concentrations compatible with a biological activity (Oteiza et al. 2018).

Hydrogen atom transfer (HAT), sequential proton loss electron transfer (SPLET) and single electron transfer followed by proton transfer (SET-PT) are mechanisms recently studied to describe thermodynamics of free radical scavenging by a set of nine isoflavones (Lengyel et al. 2013). In another recent study, kinetics of inactivation of HOO by a set of 6 isoflavones via formal HAT (fHAT) and single electron transfer (SET) has been investigated (Caicedo et al, 2014). In HAT mechanism one electron and one proton transfer as a single entity, i.e., as a hydrogen atom. Proton coupled electron transfer (PCET) mechanism involves one proton and one electron transfer from different locations in the molecule. In the case where it is not possible to clearly distinguish HAT from PCET mechanism, then the mechanism is labeled as fHAT (Galano and Alvarez-Idaboy 2019). In all mentioned mechanisms phenolic -OH group (phenoxide ion- $\mathrm{O}^{-}$) of isoflavones is considered as the active site for $\mathrm{H}$-atom, $\mathrm{H}^{+}$and/or $\mathrm{e}^{-}$ donation to a free radical, as generally is the case in flavonoid antiradical action (Zhang and $\mathrm{Ji}$ 2006).

Occasionally, an assertion can be find in litertature stating that not phenolic but C-ring hydrogens of catechins and flavanonols are responsible for antiradical activity (Kondo et al. 1999, Vo et al. 2019). Such claims were mainly based on the calculated bond dissociation enthalpy (BDE) values of $\mathrm{C}-\mathrm{H}$ and $\mathrm{O}-\mathrm{H}$ bond breaking. Position with the lowest BDE value was assumed as the active site of $\mathrm{H}$-atom abstraction because the lower the enthalpy necessary for breaking the donor- $\mathrm{H}$ bond, the higher the antiradical potency. In this way, Kondo et al. (1999) stated that $\mathrm{C} 2-\mathrm{H}$ allylic hydrogen of epicatechin $(\mathrm{BDE}=64.8 \mathrm{kcal} / \mathrm{mol})$ and epigallocatechin $(\mathrm{BDE}=64.5 \mathrm{kcal} / \mathrm{mol})$ are operative in free radical inactivation rather than corresponding $\mathrm{C} 4$ ' $-\mathrm{OH}$ phenolic hydrogen $(\mathrm{BDE}=72.1$ and $68.4 \mathrm{kcal} / \mathrm{mol}$, respectively). Similarly, because of lower $\mathrm{C}-\mathrm{H}$ BDE values by comparison with $\mathrm{O}-\mathrm{H}$ BDE values, and additional kinetic calculations, Vo et al. (2019) indicated C3-H hydrogen of dihydrokaempferol, dihydromyricetin, keto-teracacidin, pinobanksin and taxifolin as preferred site of free radical inactivation. The same was claimed for $\mathrm{C} 2-\mathrm{H}$ allylic hydrogen of naringenin and pinocembrin (Vo et al. 2019). Most of the mentioned flavonoids possess catechol moiety (two vicinal -OH groups) in A-ring or B-ring. Because of this, keeping in mind well known Bors' criteria for effective free radical scavenging (Bors et al. 1990), such statements at first glance sound 
questionable. Our suspicion is highly supported by the fact that $\mathrm{O}-\mathrm{H}$ bonds undergo fHAT faster than $\mathrm{C}-\mathrm{H}$ bonds of similar strength (Mayer 2011).

Equol is a particularly suitable molecule to reinvestigate statements that not phenolic but Cring hydrogens are responsible for antiradical activity. Equol possesses five hydrogens in C-ring structure that may compete with two phenolic hydrogens in free radical scavenging (Fig. 1).
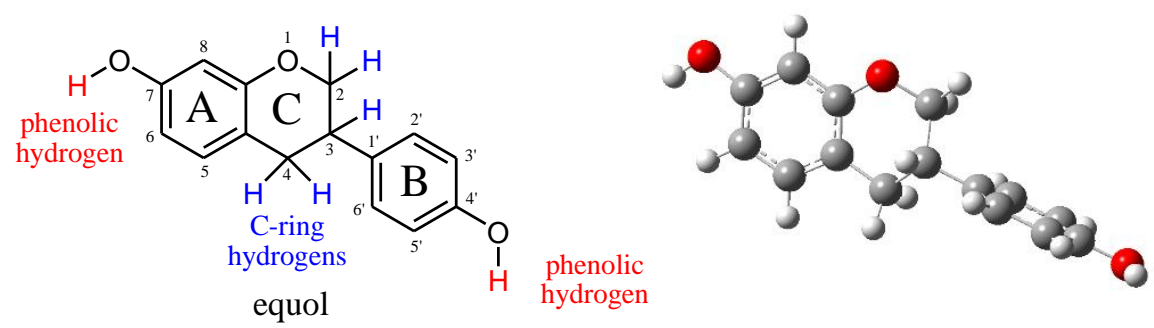

Fig. 1. Structure of equol

In this report hydrogen donation potency of equol C-ring and phenolic -OH groups to hydroperoxyl radical (HOO') was estimated by both thermodynamic and kinetic approach.

\section{Computational details}

All electronic calculations were performed using the Gaussian 09 program package (Frisch et al. 2009). Geometry optimizations and frequency calculations were carried out using the M062X hybrid meta exchange-correlation functional (Zhao and Truhlar 2008) in conjunction with the 6-311++G(d,p) basis set. The M06-2X accurately predicts chemical reaction barrier heights and thermodynamic quantities (Mardirossian and Head-Gordon 2016). The influence of solvents was calculated with an implicit continuum solvation model, SMD (Marenich et al. 2009). In conjunction with the M06-2X density functional, SMD model has been successfully used for study of thermodynamics and kinetics of free radical scavenging mechanisms (Galano and Alvarez-Idaboy 2014, Galano et al. 2016). Spin unrestricted calculations were used for open-shell systems. Local minima and transition states (TSs) were identified by the number of imaginary frequencies ( 0 and 1 , respectively). Intrinsic reaction coordinate (IRC) calculation was performed on both sides of the TS to confirm that it properly connects with the corresponding reactants and products. All computations were performed in gas-phase, pentyl ethanoate and water at $298.15 \mathrm{~K}$. BDE related to $\mathrm{H}$-atom donation was calculated as described elsewhere (Amić et al. 2017).

The rate constants $(k)$ for fHAT reactions were calculated at the M06-2X/6-311++G(d,p) level by using the conventional transition state theory (TST) as implemented in Eyringpy program (Dzib et al. 2018):

$$
k=\sigma \kappa \frac{k_{B} T}{h} e^{-\left(\Delta G^{\ddagger}\right) / R T}
$$

$\sigma$ is the reaction path degeneracy, i.e., the number of different but equivalent reaction pathways that are possible, $\kappa$ accounts for tunneling corrections calculated through Eckart and Wigner approach, $k_{\mathrm{B}}$ is the Boltzmann constant, $T$ is the temperature, $h$ is the Planck constant, and $\Delta G^{f}$ is the Gibbs free energy of activation of the studied reaction. 
For the SET reactions, the Marcus theory was used (Marcus 1997). It relies on the transition state formalism and allows calculating the barrier of any SET reaction from two thermodynamic parameters, the free energy of reaction, $\Delta G_{S E T}^{0}$, and the nuclear reorganization energy, $\lambda$ :

$$
\begin{gathered}
\Delta G_{S E T}^{\neq}=\frac{\lambda}{4}\left(1+\frac{\Delta G_{S E T}^{0}}{\lambda}\right)^{2} \\
\lambda \approx \Delta E_{S E T}-\Delta G_{S E T}^{0}
\end{gathered}
$$

$\triangle E_{S E T}$ is the nonadiabatic energy difference between reactants and vertical products for SET (Martinez et al. 2012). Accordingly, the TST rate constant for SET reactions is computed in Eyringpy program using equation:

$$
k_{S E T}=\frac{k_{B} T}{h} e^{-\left(\Delta G_{S E T}^{ \pm}\right) / R T}
$$

The branching ratios $(\Gamma)$ calculated from the rate constants can be used to identify the reaction pathways most contributing to the total reaction (in \%) (Galano and Alvarez-Idaboy 2014). They are calculated as:

$$
\Gamma=100 \frac{k_{i}}{k_{\text {overall }}}
$$

where $k_{i}$ represents the rate constant of independent path. The overall rate constant $\left(k_{\text {overall }}\right)$ is calculated as the sum of rate constants of all reaction paths.

\section{Results and discussion}

\subsection{Estimation of C-ring vs phenolic hydrogen abstraction potency}

Results of the performed thermodynamic and kinetic calculations at M06-2X/6-311++G(d,p) level of theory are summarized in Table 1. The M06-2X functional was chosen because the very widely used B3LYP functional is confirmed to systematically underestimate both BDE and barrier heights (Feng et al. 2003, Zhao et al. 2005). To achieve reliable results the more appropriate functional, such as M06-2X, should be applied in antioxidant thermodynamic and kinetic calculations (Galano and Alvarez-Idaboy 2014). For the purpose of comparison, in Table 1 the gas-phase results obtained by the M05-2X functional were included, which is widely used in kinetic investigations of free radical scavenging (Galano and Alvarez-Idaboy 2019).

By considering only thermodynamic parameters listed in Table 1, it appears that among five H-donor sites, C-4 hydrogen is the most abstractable due to the lowest BDE and $\Delta_{\mathrm{r}} G$ values. As aforementioned, the similar assumption, that $\mathrm{C}$-ring hydrogens are more abstractable than phenolic ones, was claimed two decades ago by Kondo et al. (1999). For prediction of BDE, they used unreliable low level of theory (PM3 method). Shortly after, using the B3LYP functional, the phenolic hydrogens of catechins were found as more abstractable than allylic ones (Zhang and Vang 2002). Recently, despite of the fact that it is recognized as inadequate for thermodynamic and kinetic calculations, Vo et al. (2019) also used the B3LYP functional. As indicated in Computational details section, in this report BDE was calculated by approach that 
nicely matches experimental BDE values of simple phenols (Amić et al. 2017). Nevertheless, any predicted BDE value could not be sole or the final determinant of chemical reactivity (Ingold and Pratt 2014).

\begin{tabular}{|c|c|c|c|c|c|c|c|c|c|}
\hline \multicolumn{10}{|c|}{ gas-phase M05-2X/6-311++G(d,p) } \\
\hline path & $\mathrm{BDE}$ & $\Delta_{\mathrm{r}} G$ & $v$ & $\Delta G^{\neq}$ & $k^{\mathrm{TST}}$ & $\kappa^{\text {Eck }}$ & $k^{\mathrm{TST} / \mathrm{Eck}}$ & $\kappa^{\mathrm{Wig}}$ & $k^{\mathrm{TST} / \mathrm{Wig}}$ \\
\hline $\mathrm{C}-2$ & 93.99 & 8.9 & -1695 & 22.7 & $7.23 \times 10^{-3}$ & 28.9 & $2.11 \times 10^{-1}$ & 3.8 & $2.77 \times 10^{-2}$ \\
\hline $\mathrm{C}-3$ & 88.15 & 1.6 & -1768 & 21.7 & $1.99 \times 10^{-2}$ & 93.1 & $1.87 \times 10^{0}$ & 4.0 & $7.83 \times 10^{-2}$ \\
\hline $\mathrm{C}-4$ & 84.63 & -0.2 & -1666 & 18.7 & $5.48 \times 10^{0}$ & 48.0 & $2.65 \times 10^{2}$ & 3.7 & $2.05 \times 10^{1}$ \\
\hline O-7 & 86.75 & 1.8 & -2023 & 16.8 & $6.63 \times 10^{1}$ & 152.0 & $1.02 \times 10^{4}$ & 5.0 & $3.43 \times 10^{2}$ \\
\hline $\mathrm{O}-4^{\prime}$ & 86.83 & 1.8 & -2095 & 17.8 & $1.39 \times 10^{1}$ & 269.2 & $3.67 \times 10^{3}$ & 5.3 & $7.23 \times 10^{1}$ \\
\hline & & & & $k_{\text {overall: }}$ & $8.57 \times 10^{1}$ & & $1.41 \times 10^{4}$ & & $4.36 \times 10^{2}$ \\
\hline
\end{tabular}

gas-phase M06-2X/6-311++G(d,p)

\begin{tabular}{|c|c|c|c|c|c|c|c|c|c|}
\hline path & $\mathrm{BDE}$ & $\Delta_{\mathrm{r}} G$ & $v$ & $\Delta G^{\neq}$ & $k^{\mathrm{TST}}$ & $\kappa^{\mathrm{Eck}}$ & $k^{\mathrm{TST} / \mathrm{Eck}}$ & $\kappa^{\mathrm{Wig}}$ & $k^{\mathrm{TST} / \mathrm{Wig}}$ \\
\hline $\mathrm{C}-2$ & 93.91 & 7.8 & -1819 & 21.2 & $8.43 \times 10^{-2}$ & 51.2 & $4.28 \times 10^{0}$ & 4.2 & $3.49 \times 10^{-1}$ \\
\hline $\mathrm{C}-3$ & 89.13 & 2.2 & -1919 & 21.8 & $1.51 \times 10^{-2}$ & 348.0 & $5.24 \times 10^{0}$ & 4.6 & $6.63 \times 10^{-2}$ \\
\hline $\mathrm{C}-4$ & 85.82 & -0.1 & -1740 & 18.0 & $1.81 \times 10^{1}$ & 70.5 & $1.26 \times 10^{3}$ & 3.9 & $7.23 \times 10^{1}$ \\
\hline O-7 & 87.31 & 1.5 & -2110 & 17.1 & $4.76 \times 10^{1}$ & 212.4 & $1.02 \times 10^{4}$ & 5.3 & $2.53 \times 10^{2}$ \\
\hline \multirow[t]{2}{*}{ O-4' } & 87.45 & 1.7 & -2201 & 17.4 & $2.77 \times 10^{1}$ & 376.1 & $1.02 \times 10^{4}$ & 5.7 & $1.57 \times 10^{2}$ \\
\hline & & & & $k_{\text {overall }}:$ & $9.35 \times 10^{1}$ & & $2.17 \times 10^{4}$ & & $4.82 \times 10^{2}$ \\
\hline
\end{tabular}

pentyl ethanoate $\mathrm{M} 06-2 \mathrm{X} / 6-311++\mathrm{G}(\mathrm{d}, \mathrm{p})$

\begin{tabular}{|c|c|c|c|c|c|c|c|c|c|}
\hline \\
\hline path & $\mathrm{BDE}$ & $\Delta_{\mathrm{r}} G$ & $v$ & $\Delta G^{\neq}$ & $k^{\mathrm{TST}}$ & $\kappa^{\text {Eck }}$ & $k^{\mathrm{TST} / \mathrm{Eck}}$ & $\kappa^{\mathrm{Wig}}$ & $k^{\mathrm{TST} / \mathrm{Wig}}$ \\
\hline $\mathrm{C}-2$ & 96.06 & 8.2 & -1795 & 22.8 & $2.4 \times 10^{-4}$ & 47.4 & $1.1 \times 10^{-2}$ & 4.1 & $9.9 \times 10^{-4}$ \\
\hline C-3 & 90.18 & 2.3 & -1882 & 22.2 & $3.3 \times 10^{-4}$ & 285.5 & $9.5 \times 10^{-2}$ & 4.4 & $1.5 \times 10^{-3}$ \\
\hline C-4 & 87.59 & 0.3 & -1732 & 20.0 & $2.8 \times 10^{-2}$ & 74.0 & $2.1 \times 10^{0}$ & 3.9 & $1.1 \times 10^{-1}$ \\
\hline O-7 & 87.48 & 0.1 & -2334 & 17.4 & $1.1 \times 10^{0}$ & 1126.1 & $1.3 \times 10^{3}$ & 6.3 & $7.2 \times 10^{0}$ \\
\hline \multirow[t]{2}{*}{ O-4' } & 87.83 & 0.6 & -2282 & 18.0 & $4.2 \times 10^{-1}$ & 612.6 & $2.6 \times 10^{2}$ & 6.1 & $2.6 \times 10^{0}$ \\
\hline & & & & $k_{\text {overall: }}$ & $1.5 \times 10^{0}$ & & $1.6 \times 10^{3}$ & & $9.9 \times 10^{0}$ \\
\hline
\end{tabular}

water $\mathrm{M} 06-2 \mathrm{X} / 6-311++\mathrm{G}(\mathrm{d}, \mathrm{p})$

\begin{tabular}{|c|c|c|c|c|c|c|c|c|c|}
\hline path & BDE & $\Delta_{\mathrm{r}} G$ & $v$ & $\Delta G^{\neq}$ & $k^{\mathrm{TST}}$ & $\kappa^{\text {Eck }}$ & $k^{\mathrm{TST} / \mathrm{Eck}}$ & $\kappa^{\mathrm{Wig}}$ & $k^{\mathrm{TST} / \mathrm{Wig}}$ \\
\hline $\mathrm{C}-2$ & 95.97 & 6.5 & -1789 & 20.8 & $7.5 \times 10^{-3}$ & 51.2 & $3.9 \times 10^{-1}$ & 4.1 & $3.1 \times 10^{-2}$ \\
\hline C-3 & 88.96 & -0.3 & -1755 & 19.9 & $1.5 \times 10^{-2}$ & 67.9 & $1.0 \times 10^{0}$ & 4.0 & $6.1 \times 10^{-2}$ \\
\hline $\mathrm{C}-4$ & 86.84 & -2.3 & -1696 & 18.1 & $6.9 \times 10^{-1}$ & 51.7 & $3.6 \times 10^{1}$ & 3.8 & $2.6 \times 10^{0}$ \\
\hline O-7 & 86.98 & -1.9 & -3059 & 17.8 & $5.8 \times 10^{-1}$ & 10581.5 & $6.2 \times 10^{3}$ & 10.1 & $5.9 \times 10^{0}$ \\
\hline \multirow[t]{2}{*}{ O-4' } & 87.51 & -1.3 & -2654 & 18.0 & $4.1 \times 10^{-1}$ & 5014.8 & $2.0 \times 10^{3}$ & 7.8 & $3.2 \times 10^{0}$ \\
\hline & & & & $k_{\text {overall }}:$ & $1.7 \times 10^{0}$ & & $8.2 \times 10^{3}$ & & $1.2 \times 10^{1}$ \\
\hline
\end{tabular}

Table 1. Bond dissociation enthalpies BDE (kcal/mol), reaction Gibbs free energies $\Delta_{\mathrm{r}} G$ (kcal/mol), TS imaginary frequencies $v\left(\mathrm{~cm}^{-1}\right)$, activation Gibbs free energies $\Delta G^{\ddagger}(\mathrm{kcal} / \mathrm{mol})$, TST $\left(k^{\mathrm{TST}}\right)$, Eckart $\left(k^{\mathrm{TST} / \mathrm{Eck}}\right)$ and Wigner $\left(k^{\mathrm{TST} / \mathrm{Wig}}\right)$ rate constants $\left(\mathrm{M}^{-1} \mathrm{~s}^{-1}\right)$ with corresponding Eckart $\left(\kappa^{\mathrm{Eck}}\right)$ and Wigner $\left(\kappa^{\mathrm{Wig}}\right)$ tunneling coefficients in fHAT reaction of equol with $\mathrm{HOO}^{\circ}$ at $298.15 \mathrm{~K}$.

Although the BDE may be an important factor governing the fHAT reactions, a more complete understanding would require kinetic analysis. It has long been known that main group 
free radicals abstract $\mathrm{H}$-atom much faster from $\mathrm{O}-\mathrm{H}$ bonds than from $\mathrm{C}-\mathrm{H}$ bonds of similar strength, showing that BDE is not the only determinant of fHAT reactivity (Mayer 2011). For instance, oxyl radicals react $\sim 10^{4}$ faster with $\mathrm{O}-\mathrm{H}$ bonds than with $\mathrm{C}-\mathrm{H}$ bonds of comparable strength (Mahoney and DaRooge 1975, Mayer et al. 2006).

The simplest approach to study the rate constants is the non-variational transition state theory (TST) (Truhlar 1983) since it requires very limited information about the potential energy surface (PES): electronic energies and frequencies of the reactants, products and transition state on the PES. Tunneling factor in hydrogen abstraction reactions is significant and must be taken into account. TST and Eckart's tunneling correction could be a good approximation for the calculation of bimolecular rate constants (Espinosa-Garcia et al. 1994). In order to achieve a better estimate of the rate constants of bimolecular $\mathrm{H}$-abstraction reactions, the reactant and product complexes are taken into account in Eyringpy program (AlvarezIdaboy et al. 2001, Dzib et al. 2018).

Amongst kinetic parameters $\left(\Delta G^{\ddagger}\right.$ and $\left.k\right)$ listed in Table 1 , the rate constants are more relevant than $\Delta G^{\neq}$to compare the different reaction paths since fHAT reactions involve tunneling effect. It was suggested that the quantum mechanical tunneling is the key factor affecting the fastness of fHAT reactions because a light particle (H-atom) can easily tunnel through the reaction barrier (Navarrete et al. 2005). Without tunneling corrections, the overall rate constant ( $k_{\text {overall }}$ ) of the reaction of equol with HOO is $\sim 10^{1} \mathrm{M}^{-1} \mathrm{~s}^{-1}$ at $298.15 \mathrm{~K}$, while by including Eckart tunneling corrections it amounts to $\sim 10^{4} \mathrm{M}^{-1} \mathrm{~s}^{-1}$ in all investigated media at the same temperature.

Both M06-2X and M05-2X functionals indicated preferred H-atom abstraction from phenolic $\mathrm{OH}$ groups of equol in comparison with $\mathrm{C}$-ring hydrogens (Table 1). Preferred fHAT from phenolic $\mathrm{C} 7-\mathrm{OH}$ and $\mathrm{C} 4{ }^{\prime}-\mathrm{OH}$ groups $\left(k^{\mathrm{TST} / \mathrm{Eck}}\right)$ in comparison with $\mathrm{C} 2-\mathrm{H}$ and $\mathrm{C} 3-\mathrm{H}$ is in line with the known fact that $\mathrm{O}-\mathrm{H}$ bonds are intrinsically much more reactive than $\mathrm{C}-\mathrm{H}$ bonds of similar strength, by a factor of $\sim 10^{4}$ (Mayer 2011). However, in the case of C4-H nearly $10^{2}$ times higher reactivity of phenolic hydrogens was found.

To additionally quantify the contributions of the C-ring hydrogens $v s$ phenolic hydrogens on the overall $\mathrm{HOO}^{*}$ scavenging potency of equol, the branching ratios $(\Gamma)$ were estimated. Because Eckart tunneling corrections are more reliable than Wigner corrections (Dzib et al. 2018) it was assumed that the branching ratios related to the Eckart approach are more appropriate to ascertain preferred reaction path(s). Data presented in Table 2 confirm that the $\mathrm{HOO}^{*}$ scavenging by equol takes place dominantly via hydrogen atom donation from phenolic $\mathrm{C} 7-\mathrm{OH}$ and $\mathrm{C} 4$ '-OH groups. Amongst C-ring hydrogens, only those from the C-4 site slightly contribute to the $\mathrm{HOO}^{\circ}$ inactivation in gas-phase.

\begin{tabular}{lccc}
\hline reaction path & gas-phase & pentyl ethanoate & water \\
\hline C2-H & 0 & 0 & 0 \\
C3-H & 0 & 0 & 0 \\
C4-H & $6(2)$ & 0 & 0 \\
C7-OH & $47(72)$ & 83 & 76 \\
C4'-OH & $47(26)$ & 17 & 24 \\
\hline
\end{tabular}

Table 2. Branching ratios $\Gamma(\%)$ related to the M06-2X (M05-2X) calculations at $298.15 \mathrm{~K}$.

Deviation from the expected $k$ value difference of $\sim 10^{4}$ in the case of $\mathrm{C} 4-\mathrm{H}$ abstraction (Table 1) may be related to the fact that the Wigner, Eckart and zero-curvature tunneling (ZCT) corrections may severely underestimate the importance of tunneling for the $\mathrm{O}-\mathrm{H}$ abstraction in comparison with the $\mathrm{C}-\mathrm{H}$ abstraction (Sun and Saeys 2008). We suppose that the more accurate 
results could be achieved by using canonical variational transition state theory (CVT) corrected by the semiclassical multidimensional small-curvature tunneling (SCT) (Bao and Truhlar 2017). The CVT/SCT method is successfully applied to the reactions of free radical scavenging by natural antioxidants (Navarrete et al. 2005, Tejero et al. 2007, Chiodo et al. 2010).

Fig. 2 shows potential energy profiles for the $\mathrm{H}$-atom transfer from the most reactive sites of equol (C7-OH, C4'-OH and $\mathrm{C} 4-\mathrm{H})$ to $\mathrm{HOO}^{\circ}$, that correspond to the optimized geometries.

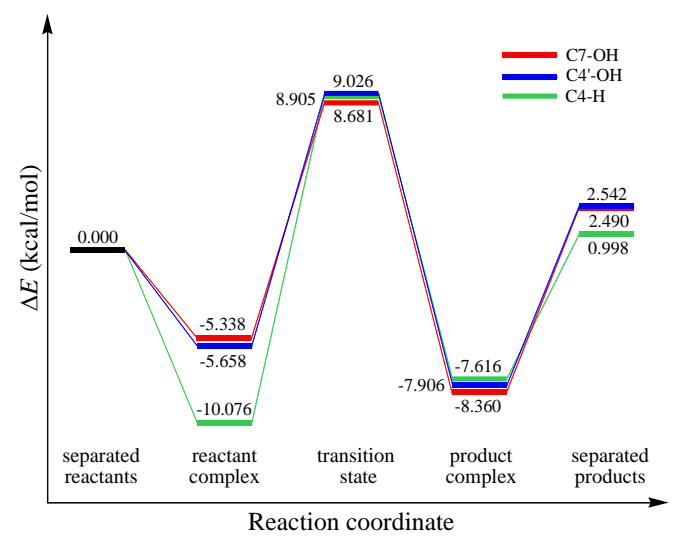

Fig. 2. Potential energy profiles for the reaction of hydroperoxyl radical with the $\mathrm{C} 7-\mathrm{OH}$ (red line), $\mathrm{C} 4$ '-OH (blue line) and $\mathrm{C} 4-\mathrm{H}$ (green line) sites of equol in gas-phase.

It should be emphasized that the product complexes are more stable than separated products, as earlier has been found in scavenging of peroxyl radicals by other naturally occurring antioxidants (Navarrete et al. 2005, Chiodo et al. 2010). The higher stability of product complexes indicates that $\mathrm{H}_{2} \mathrm{O}_{2}$ product remains bound to the equol free radical for a while. In this way, pro-oxidant activity of equol (oxidation by the equol's free radicals of the target assumed to be protected) and $\mathrm{H}_{2} \mathrm{O}_{2}$ is reduced.

\subsection{Evaluation of the reliability of calculated results}

The preferred way to ascertain reliability of applied theoretical model is comparison of obtained results with related experimental findings. To the best of our knowledge there is no available experimental kinetic results of $\mathrm{HOO}^{*}$ inactivation by equol. Because of that, we performed kinetic calculations for well-known extensively studied natural antioxidant $\alpha$-tocopherol and its water soluble synthetic derivative trolox, and compared obtained results with published experimental as well as theoretical results (Table 3).

Rate constant values for reaction of $\alpha$-tocopherol and its structural analogs with ROO` in solvents of different polarity have been experimentally assayed (Bielski et al. 1985, Burton et al. 1985, Cedrowski et al. 2016). In aqueous solution of $0.022 \mathrm{M}$ sulfuric acid in $85 \%$ ethanol, $k$ value of $2.00 \times 10^{5} \mathrm{M}^{-1} \mathrm{~s}^{-1}$ was obtained for inactivation of HOO by $\alpha$-tocopherol (Bielski et al. 1985). It could be presumed that in such acidic media deprotonation of phenolic OH group of $\alpha$-tocopherol $\left(\mathrm{p} K_{\mathrm{a}}=11.92\right.$ ) is suppressed, thus eliminating SPLET to leave only fHAT mechanism (Musialik and Litwinienko 2005). The size of a considered $\alpha$-tocopherol molecule may be reduced by replacing a long hydrophobic phytyl tail by methyl group obtaining 2,2,5,7,8-pentamethyl-6-chromanol (TOH). It has been shown that $k$ values are negligibly influenced by size of attached chain: in nonpolar medium, for inactivation of $\mathrm{HOO}^{\circ}$ by $\alpha$ tocopherol, TOH and TOH1, experimental $k$ values of $3.2 \times 10^{6} \mathrm{M}^{-1} \mathrm{~s}^{-1}, 3.8 \times 10^{6} \mathrm{M}^{-1} \mathrm{~s}^{-1}$ and 
$2.7 \times 10^{6} \mathrm{M}^{-1} \mathrm{~s}^{-1}$, respectively, were obtained (Burton et al. 1985). Using CVT/SCT methodology in calculation the barrier heights and tunneling corrections, previously theoretically calculated rate constant for $\alpha$-tocopherol TOH1 analogue, in the gas-phase, was $1.5 \times 10^{5} \mathrm{M}^{-1} \mathrm{~s}^{-1}$ (Navarrete et al. 2005). TOH1 is $\alpha$-tocopherol analogue that bears $\mathrm{H}$-atoms on $\mathrm{C}-2$ atom (instead of methyl groups in $\mathrm{TOH}$ ).

Equal or very similar rate orders have been found for reaction of $\mathrm{TOH}$ with styrylperoxyl radical (Cedrowski et al. 2016). It has been suggested that structure of peroxyl radicals mostly has no significant impact on the $k$ values of $\mathrm{H}$-atom abstraction (Burton et al. 1985, Cedrowski et al. 2016). This fact should be emphasized because results of Burton et al. (1985) were obtained by scavenging of styrylperoxyl radical.

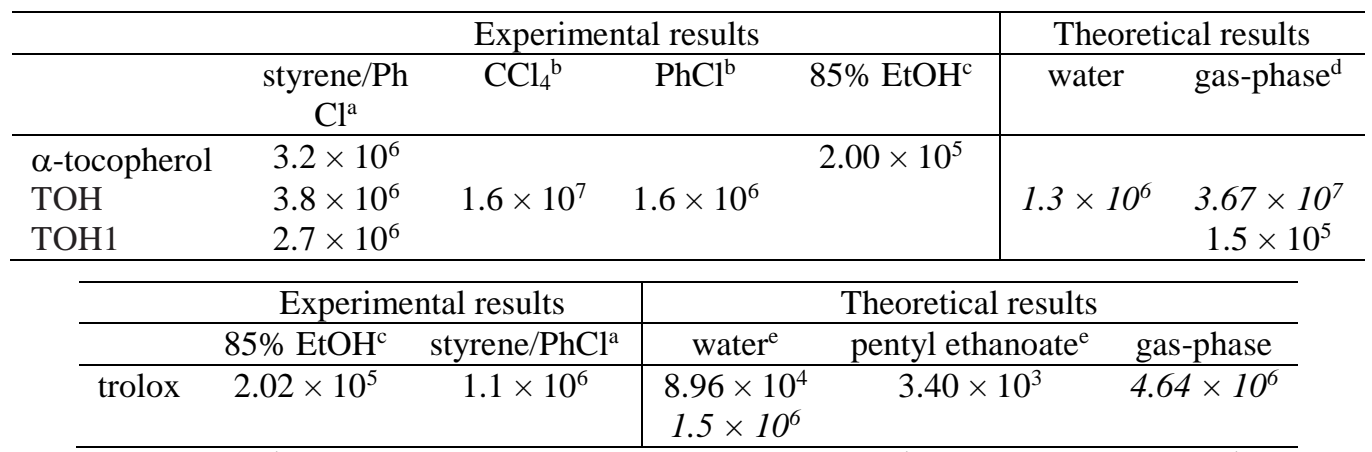

${ }^{\mathrm{a}}$ Burton al. 1985; ${ }^{\mathrm{b}}$ Cedrowski et al. 2016; ${ }^{\mathrm{c}}$ Bielski et al 1985; ${ }^{\mathrm{d}}$ Navarrete et al. 2005; ${ }^{\mathrm{d}}$ Bielski et al 1985; ${ }^{\text {e }}$ Alberto et al. 2013; our results are given in italics

Table 3. Experimental and calculated rate constants $k$ (in $\mathrm{M}^{-1} \mathrm{~s}^{-1}$ ) for bimolecular inactivation of peroxyl radical by $\alpha$-tocopherol (and its structural analogues $\mathrm{TOH}$ and $\mathrm{TOH} 1$ ) and trolox.

We suppose that the results given in Table 3 could be mutually roughly compared because of the same underlying mechanism (fHAT). By inspection of the data presented in Table 3, it could be concluded that here applied theoretical approach seems reasonable because our results (given in italics) fairly match experimental results obtained in different media. The obtained results presented in Table 1 indicate that equol reacts with $\mathrm{HOO}^{*}$ slower than $\alpha$-tocopherol and its structural analogues by approximately two orders of magnitude (Table 3). Equol also appears as less potent $\mathrm{HOO}^{\circ}$ scavenger than trolox.

\subsection{Estimation of preferred fHAT mechanism}

The simplest method of distinction between the HAT and PCET mechanisms is based on the character of the singly occupied molecular orbital (SOMO) at the transition state (Mayer et al. 2002). According to this method, the transfer of equol's C-ring hydrogens to HOO occurs via HAT mechanism, as illustrated for the abstraction of the $\mathrm{C} 4$ hydrogen in Fig. 3a. In transition state (TS) structure for this reaction, the SOMO has significant density in orbitals that lie along the $\mathrm{C}$-donor $\cdots \mathrm{H} \cdots \mathrm{O}$-acceptor axis and the transferring moiety between $\mathrm{C}$ and $\mathrm{O}$ atoms is a hydrogen atom.

The transfer of phenolic hydrogens to HOO` occurs via PCET mechanism (Fig. 3b): the SOMO of PCET TSs involve $\pi$ orbitals that are orthogonal to the O-donor $\cdots \mathrm{H} \cdots \mathrm{O}$-acceptor vector. Proton is transferred between lone pairs of electrons in $\sigma$ orbitals on the oxygens and an electron is synchronously transferred from the doubly occupied $\pi$ orbital on the oxygen of equol $7-\mathrm{OH}$ group to the singly occupied $\pi$ orbital on the oxygen of $\mathrm{HOO}^{*}$. The obtained results are 
consistent with the published ones regarding different reactivity of $\mathrm{O}-\mathrm{H} v s \mathrm{C}-\mathrm{H}$ bonds: phenolic $\mathrm{O}-\mathrm{H}$ bonds followed the PCET and $\mathrm{C}-\mathrm{H}$ bonds the HAT mechanism (Mayer et al. 2002, Galano et al. 2010, Usharani et al. 2013).

a)
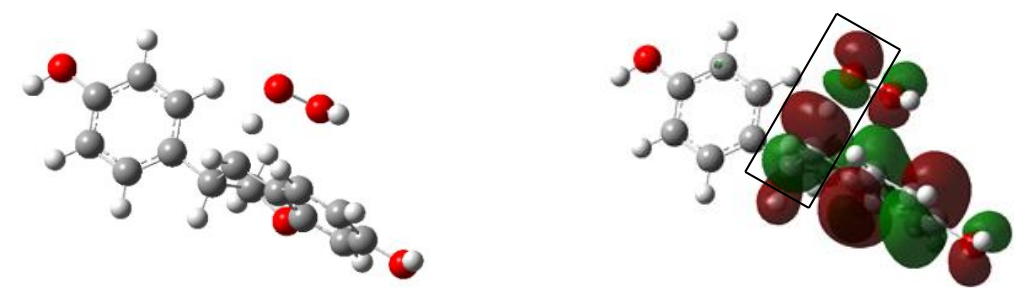

b)
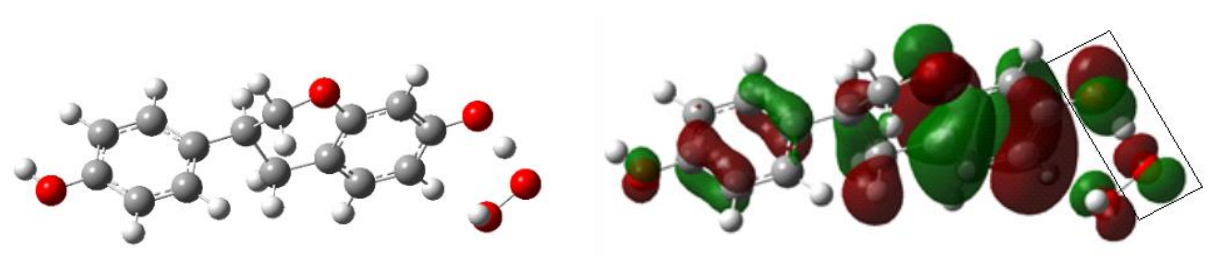

Fig. 3. TS structure of the reaction of equol with $\mathrm{HOO}^{*}$ accompanied by the corresponding SOMO: a) abstraction of $\mathrm{C} 4$ hydrogen; b) abstraction of phenolic $\mathrm{C} 7-\mathrm{OH}$ hydrogen.

Fig. 3 also illustrates the advance of $\mathrm{H}$-atom transfer between two heteroatoms (Inagaki et al. 2014). $\mathrm{HOO}^{\circ}$ abstracts $\mathrm{H}$-atom from the $\mathrm{O}-\mathrm{H}$ donor group faster than from the $\mathrm{C}-\mathrm{H}$ donor group mainly because of the presence of an H-bonded complex between the reactants (TS donor-acceptor distance $2.34 \AA$ ) , thus favoring PCET mechanism (Ingold and Pratt 2014). In reaction of the $\mathrm{C}-\mathrm{H}$ group, hydrogen bonding is less pronounced (TS donor-acceptor distance $2.54 \AA$ ).

\subsection{Equol's potency to protect from hydroperoxyl radical damage}

In order to predict if equol is efficient as a free radical scavenger, reaction with the hydroperoxyl radical is particularly suitable because it is the simplest of the biologically relevant peroxyl radicals (ROO') with intermediate reactivity and not too short half-live to be effectively scavenged by phenolic compounds (Galano and Alvarez-Idaboy 2019). Hydroperoxyl radical can initiate peroxidation of fatty acids resulting in membrane lipid damage (Aikens and Dix 1991). Oxidative damage to lipids may underlay degenerative diseases and aging.

The experimentally measured rate constants corresponding to the reactions of $\mathrm{HOO}^{\bullet}$ with polyunsaturated fatty acids (PUFAs) are in the range $1.18-3.05 \times 10^{3} \mathrm{M}^{-1} \mathrm{~s}^{-1}$, assayed in aqueous ethanolic solutions at very low pH (Bielski et al. 1983). Compounds that react faster with $\mathrm{HOO}^{\circ}$ than the double allylic $\mathrm{H}$-atom of the PUFAs are expected to act as efficient antioxidants (Galano 2015).

Here obtained result in water solution $\left(k_{\text {overall }}=8.2 \times 10^{3} \mathrm{M}^{-1} \mathrm{~s}^{-1}\right)$ indicates equol as efficient protector from $\mathrm{HOO}^{*}$ damage via fHAT mechanism. To ascertain equol's potency to inactivate $\mathrm{HOO}^{\circ}$ via SET mechanism, $k_{\mathrm{SET}}$ for single electron transfer from equol $4^{\prime}-\mathrm{O}^{-}$ 
phenoxide anion to $\mathrm{HOO}^{\bullet}$ in water as a solvent was calculated. For two $\mathrm{p} K_{\mathrm{a}}$ values of equol, the value $\mathrm{p} K_{\mathrm{a}}=9.84$ is considered to be common for the two hydroxyls, taking equol as a monoprotic acid (Han et al. 2009). Despite the fact that the molar fraction of equol monoanion is very low (0.004) at physiological $\mathrm{pH}$, its reactivity dominates the overall rate constant (Caicedo et al. 2014). For this reaction, by including the molar fraction of $\mathrm{HOO}^{\circ}$ at $\mathrm{pH}=7.4$, the obtained value of $k_{\mathrm{SET}}=1.40 \times 10^{6} \mathrm{M}^{-1} \mathrm{~s}^{-1}\left(\Delta G_{S E T}^{0}=3.2 \mathrm{kcal} / \mathrm{mol} ; \Delta G_{S E T}^{\neq}=5.5 \mathrm{kcal} / \mathrm{mol} ; \lambda=\right.$ 15.0) was in good agreement with previously published: $k_{\mathrm{SET}}=7.60 \times 10^{5} \mathrm{M}^{-1} \mathrm{~s}^{-1} ; \Delta G_{S E T}^{\neq}=$ $6.15 \mathrm{kcal} / \mathrm{mol} ; \lambda=27.8$ (Caicedo et al. 2014). Therefore, equol is expected as able for preventing peroxyl oxidation of lipids via SET because it reacts faster with HOO` than bisallylic hydrogen in PUFAs. This prediction is in line with in vivo results that the consumption of soy increases resistance of low-density lipoprotein to oxidation in humans (Wiseman et al. 2000), and in vitro inhibition of LDL oxidation and membrane lipid peroxidation by equol (Hwang et al. 2000), where free radical scavenging may take a part in protecting activity. Equol also has potential to react faster with $\mathrm{HOO}^{\circ}$ than proteins and DNA, because reactivity of these biological targets is lower than that of bisallylic hydrogens in PUFAs (Villuendas-Rey et al. 2015). Hence, obtained kinetic data imply that equol has potential to protect biologically important molecules against $\mathrm{HOO}^{\circ}$ induced oxidative damage.

Data presented in Table 4 indicate that in gas-phase daidzein (equol precursor) reacts slower with HOO via fHAT $\left(k=6.13 \times 10^{3} \mathrm{M}^{-1} \mathrm{~s}^{-1}\right)$ than equol $\left(k=2.17 \times 10^{4} \mathrm{M}^{-1} \mathrm{~s}^{-1}\right)$. This is in accordance with experimental results of antioxidant and free radical scavenging activity of soy isoflavones and their metabolites (Wiseman et al. 2000, Rimbach et al. 2003) and with theoretical predictions (Caicedo et al. 2014). In a liposome model system, greater flexibility for conformational changes of equol structure enables easier penetration into the interior of the membrane than more rigid structure of its precursor daidzein (Arora et al. 1998). This contribute to equol's higher antioxidant activity in comparison with daidzein (Hodgson et al. 1996, Mitchell et al. 1998).

\begin{tabular}{lrrrrccccc}
\hline path & \multicolumn{1}{c}{ BDE } & \multicolumn{1}{c}{$\Delta_{\mathrm{r}} G$} & \multicolumn{1}{c}{$v$} & $\Delta G^{\ddagger}$ & $k^{\mathrm{TST}}$ & $\kappa^{\text {Eck }}$ & $k^{\mathrm{TST} / \mathrm{Eck}}$ & $\kappa^{\mathrm{Wig}}$ & $k^{\mathrm{TST} / \mathrm{Wig}}$ \\
\hline O-7 & 108.24 & 22.6 & -2287 & 20.4 & $1.81 \times 10^{-1}$ & 624.8 & $1.14 \times 10^{2}$ & 6.1 & $1.08 \times 10^{0}$ \\
O-4 & 86.73 & 1.0 & -2146 & 17.5 & $2.11 \times 10^{1}$ & 295.7 & $6.02 \times 10^{3}$ & 5.5 & $1.14 \times 10^{2}$ \\
\hline \multicolumn{8}{r}{} & \multicolumn{8}{c}{$k_{\text {overall }}:$} & $2.12 \times 10^{1}$ & & $6.13 \times 10^{3}$ & & $1.15 \times 10^{2}$ \\
\hline
\end{tabular}

Table 4. Bond dissociation enthalpies BDE (kcal/mol), reaction Gibbs free energies $\Delta_{\mathrm{r}} G$ (kcal/mol), TS imaginary frequencies $v\left(\mathrm{~cm}^{-1}\right)$, activation Gibbs free energies $\Delta G^{\ddagger}(\mathrm{kcal} / \mathrm{mol})$, TST $\left(k^{\mathrm{TST}}\right)$, Eckart $\left(k^{\mathrm{TST} / \mathrm{Eck}}\right)$ and Wigner $\left(k^{\mathrm{TST} / \mathrm{Wig}}\right)$ rate constants $\left(\mathrm{M}^{-1} \mathrm{~s}^{-1}\right)$ with corresponding Eckart $\left(\kappa^{\mathrm{Eck}}\right)$ and Wigner $\left(\kappa^{\mathrm{Wig}}\right)$ tunneling coefficients in fHAT reaction of daidzein with ${ }^{\circ} \mathrm{OOH}$ in gas-phase at $298.15 \mathrm{~K}$.

The results presented in this article can be compared with previously published ones dealing with $\mathrm{HOO}^{\bullet}$ trapping by isoflavones. By using SMD/M05-2X/6-311++G(d,p) level of theory accompanied with classical TST/ZCT method, Caicedo et al. (2014) obtained $k=1.75 \times$ $10^{3} \mathrm{M}^{-1} \mathrm{~s}^{-1}$ and $k=1.83 \times 10^{2} \mathrm{M}^{-1} \mathrm{~s}^{-1}$ for the fHAT reaction of equol with HOO in water and pentyl ethanoate, respectively. Deviations in relation to our results presented in Table $1(k=8.2$ $\times 10^{3} \mathrm{M}^{-1} \mathrm{~s}^{-1}$ and $k=1.6 \times 10^{3} \mathrm{M}^{-1} \mathrm{~s}^{-1}$ in water and pentyl ethanoate, respectively) could be mainly related to applied tunneling corrections which may have high impact on calculated $k$ values. For example, in $\mathrm{H}$-atom abstraction from $\alpha$-tocopherol by $\mathrm{HOO}^{*}$ in gas-phase, about $80 \%$ of the fHAT reactivity at $298.15 \mathrm{~K}$ is tunneling contribution estimated by using CVT/SCT method (Navarrete et al. 2005). Recalling that the Wigner, Eckart and ZCT corrections may severely underestimate the importance of tunneling for the $\mathrm{O}-\mathrm{H}$ abstraction (Sun and Saeys 
2008), it appears that a more sophisticated methodology (CVT/SCT) is needed for better estimation of equol's potency to scavenge HOO` via fHAT mechanism.

\section{Conclusions}

Performed electronic structure and kinetic calculations at M06-2X/6-311++G(d,p) level of theory, accompanied by the TST and Eckart tunneling corrections, indicated phenolic hydrogens of equol as much more abstractable than C-ring hydrogens. Thus, the C-ring hydrogens are poor $\mathrm{HOO}^{*}$ scavengers with negligible contribution to equol antioxidant potency. This is in line with the known facts related to phenolic $\mathrm{O}-\mathrm{H}$ vs $\mathrm{C}-\mathrm{H}$ reactivity and structural requirements needed for antioxidant activity of flavonoids, but opposite to recently published predictions.

Acknowledgements: The authors are grateful to the Croatian Ministry of Science and Education, and the Ministry of Education, Science and Technological Development of the Republic of Serbia.

\section{References}

Aikens J, Dix TA (1991). Perhydroxyl radical (HOO') initiated lipid peroxidation. The role of fatty acid hydroperoxydes. J. Biol. Chem., 266, 15091-15098.

Alberto ME, Russo N, Grand A, Galano A (2013). A physicochemical examination of the free radical scavenging activity of Trolox: mechanism, kinetics and influence of the environment. Phys. Chem. Chem. Phys., 15, 4642-4650.

Alvarez-Idaboy JR, Mora-Diez N, Boyd RJ, Vivier-Bunge A (2001). On the importance of prereactive complexes in molecule-radical reactions: Hydrogen abstraction from aldehydes by OH. J. Am. Chem. Soc., 2001, 123, 2018-2024.

Amić A, Lučić B, Stepanić V, Marković Z, Marković S, Dimitrić Marković JM, Amić D (2017). Free radical scavenging potency of quercetin catecholic colonic metabolites: Thermodynamics of $2 \mathrm{H}^{+} / 2 \mathrm{e}^{-}$processes. Food Chem., 218, 144-151.

Arora A, Nair MG, Strasburg GM (1998). Antioxidant activities of isoflavones and their biological metabolites in a liposomal system. Arch. Biochem. Biophys., 356, 133-141.

Bao JL, Truhlar DG (2017). Variational transition state theory: theoretical framework and recent developments. Chem. Soc. Rev., 46, 7548-7596.

Bielski BHJ, Arudi RL, Sutherland MW (1983). A study of the reactivity of $\mathrm{HO}_{2} / \mathrm{O}_{2}{ }^{-}$with unsaturated fatty acids. J. Biol. Chem., 258, 4759-4761.

Bielski BHJ, Cabelli DE, Arudi RL (1985). Reactivity of $\mathrm{HO}_{2} / \mathrm{O}_{2}{ }^{-}$radicals in aqueous solution. J. Phys. Chem. Ref. Data, 14, 1041-1100.

Bors W, Heller W, Michel C, Saran M (1990). Flavonoids as antioxidants: Determination of radical-scavenging efficiencies, in: Packer L, Glazer AN (Eds.), Methods in Enzymology, Academic Press, San Diego, Vol. 186, pp. 343-355.

Burton GW, Doba T, Gabe EJ, Hughes L, Lee FL, Prasad L, Ingold KU (1985). Autoxidation of biological molecules. 4. Maximizing the antioxidant activity of phenols. J. Am. Chem. Soc., 107, 7053-7065.

Caicedo C, Iuga C, Castaneda-Arriaga R, Alvarez-Idaboy JR (2014). Antioxidant activity of selected natural polyphenolic compounds from soybean via peroxyl radical scavenging. RSC Adv., 4, 38918-38930. 
Cedrowski J, Litwinienko G, Baschieri A, Amorati R (2016). Hydroperoxyl radicals (HOO*): Vitamin $\mathrm{E}$ regeneration and H-bond effects on the hydrogen atom transfer. Chem. Eur J., 22, 16441-16445.

Chiodo SG, Leopoldini M, Russo N, Toscano M (2010). The inactivation of lipid peroxide radical by quercetin. A theoretical insight. Phys. Chem. Chem. Phys., 12, 7662-7670.

Choi EJ (2009). Evaluation of equol function on anti- or prooxidant status in vivo. J. Food Sci., 74, H65-H71.

Dzib E, Cabellos JL, Ortiz-Chi F, Pan S, Galano A, Merino G (2018). Eyringpy: A program for computing rate constants in the gas phase and in solution. Int. J. Quantum Chem., 119, e25686.

Espinosa-Garcia J, Olivares del Valle FJ, Corchado JC (1994). Transition state theory and Eckart's tunneling factor: a good approximation for the calculation of bimolecular rate constants? Chem. Phys., 183, 95-100.

Feng Y, Liu L, Wang J-T, Huang H, Guo Q-X (2003). Assessment of experimental bond dissociation energies using composite ab initio methods and evaluation of the performances of density functional methods in the calculation of bond dissociation energies. J. Chem. Inf. Comput. Sci., 43, 2005-2013.

Finkel T, Holbrook NJ (2000). Oxidants, oxidative stress and the biology of ageing. Nature, 408, 239-247.

Frisch MJ, Trucks GW, Schlegel HB et al. (2009). Gaussian 09 Revision A.02, Gaussian Inc., Wallingford, CT.

Galano A (2015). Free radicals induced oxidative stress at a molecular level: The current status, challenges and perspectives of computational chemistry based protocols. J. Mex. Chem. Soc., 59, 231-262.

Galano A, Alvarez-Idaboy JR (2014). Kinetics of radical-molecule reactions in aqueous solution: A benchmark study of the performance of density functional methods. J. Comput. Chem., 35, 2019-2026.

Galano A, Alvarez-Idaboy JR (2019). Computational strategies for predicting free radical scavengers' protection against oxidative stress: Where are we and what might follow? Int. J. Quantum Chem., 119, e25665.

Galano A, Macias-Ruvalcaba NA, Medina Campos ON, Pedraza-Chaverri J (2010). Mechanism of the $\mathrm{OH}$ radical scavenging activity of nordihydroguaiaretic acid: A combined theoretical and experimental study. J. Phys. Chem. B, 114, 6625-6635.

Galano A, Mazzone G, Alvarez-Diduk R, Marino T, Alvarez-Idaboy JR, Russo N (2016). Food antioxidants: Chemical insights at the molecular level. Annu. Rev. Food Sci. Technol., 7, 335-352.

Galleano M, Verstraeten SV, Oteiza PI, Fraga CG (2010). Antioxidant actions of flavonoids: Thermodynamic and kinetic analysis. Arch. Biochem. Biophys., 501, 23-30.

Han R-M, Tian Y-X, Liu Y, Chen C-H, Ai X-C, Zhang J-P, Skibsted LH (2009). Comparison of flavonoids and isoflavonoids as antioxidants. J. Agric. Food Chem., 57, 3780-3785.

Hodgson JM, Croft KD, Puddey IB, Mori TA, Beilin LJ (1996). Soybean isoflavonoids and their metabolic products inhibit in vitro lipoprotein oxidation in serum. J. Nutr. Biochem., 7, 664-669.

Hounsome N, Hounsome B, Tomos D, Edward-Jones G (2009). Changes in antioxidant compounds in white cabbage during winter storage. Postharvest Biol. Tec. 52, 173-179.

Hwang J, Sevanian A, Hodis HN, Ursini F (2000). Synergistic inhibition of LDL oxidation by phytoestrogens and ascorbic acid. Free Radic. Biol. Med., 29, 79-89.

Inagaki T, Yamamoto T (2014). Critical role of deep hydrogen tunneling to accelerate the antioxidant reaction of ubiquinol and vitamin E. J. Phys. Chem. B, 118, 937-950.

Ingold KU, Pratt DA (2014). Advances in radical-trapping antioxidant chemistry in the $21^{\text {st }}$ century: A kinetics and mechanisms perspective. Chem. Rev., 114, 9022-9046. 
Jackson RL. Greiwe JS, Schwen RJ (2011). Emerging evidence of the health benefits of Sequol, an estrogen receptor $\beta$ agonist. Nutr. Rev. 69, 432-448.

Kondo K, Kurihara M, Miyata N, Suzuki T, Toyoda M (1999). Mechanistic studies of catechins as antioxidants against radical oxidation. Arch. Biochem. Biophys., 362, 79-86.

Lengyel J, Rimarčik J, Vaganek A, Klein E (2013). On the radical scavenging activity of isoflavones: thermodynamics of $\mathrm{O}-\mathrm{H}$ bond cleavage. Phys. Chem. Chem. Phys., 15, 1089510903.

Li B-J (2019). Advances in exploring equol production and application. J. Food Process. Preserv., 43, e14205.

Mardirossian N, Head-Gordon M (2016). How accurate are the Minnesota density functionals for noncovalent interactions, isomerization energies, thermochemistry, and barrier heights involving molecules composed of main-group elements? J. Comput. Theor. Chem., 12, 4303-4325.

Mahoney LR, DaRooge MA (1975). The kinetic behavior and thermochemical properties of phenoxy radicals. J. Am. Chem. Soc., 97, 4722-4731.

Marcus RA (1997). Electron transfer reactions in chemistry. Theory and experiment. Pure Appl. Chem., 69, 13-29.

Marenich AV, Cramer CJ, Truhlar DG (2009). Universal solvation model based on solute electron density and on a continuum model of the solvent defined by the bulk dielectric constant and atomic surface tensions. J. Phys. Chem. B, 113, 6378-6396.

Martinez A, Hernandez-Marin E, Galano A (2012). Xanthones as antioxidants: A theoretical study on the thermodynamics and kinetics of the single electron transfer mechanism. Food Funct., 3, 442-450.

Mayer JM (2011). Understanding hydrogen atom transfer: From bond strengths to Marcus theory. Acc. Chem. Res., 44, 36-46.

Mayer JM, Hrovat DA, Thomas JL, Borden WT (2002). Proton-coupled electron transfer versus hydrogen atom transfer in benzyl/toluene, methoxyl/methanol, and phenoxyl/phenol selfexchange reactions. J. Am. Chem. Soc., 124, 11142-11147.

Mayer JM, Mader EA, Roth JP, Bryant JR, Matsuo T, Dehestani A, Bales BC, Watson EJ, Osako T, Valliant-Saunders K, Lam WH, Hrovat DA, Thatcher Borden W, Davidson ER (2006). Stoichiometric oxidations of $\sigma$-bonds: Radical and possible non-radical pathways. J. Mol. Catal. A: Chem., 251, 24-33.

Mayo B, Vazquez L, Florez AB (2019). Equol: A bacterial metabolite from the daidzein isoflavone and its presumed beneficial health effects. Nutrients, 11, 2231.

Mitchell JH, Gardner PT, McPhail DB, Morrice PC, Collins AR, Duthie GG (1998). Antioxidant efficacy of phytoestrogens in chemical and biological model systems. Arch. Biochem. Biophys., 360, 142-148.

Musialik M, Litwinienko G (2005). Scavenging of dpph radicals by vitamin E is accelerated by its partial ionization: the role of sequential proton loss electron transfer. Org. Lett., 7, 49514954.

Mustonen EA, Tuori M, Saastamoinen I, Taponen J, Wahala K, Saloniemi H, Vanhatalo A (2009). Equol in milk of dairy cows is derived from forage legumes such as red clover. $\mathrm{Br}$. J. Nutr., 102, 1552-1556.

Navarrete M, Rangel C, Espinoza-Garcia J, Corchado JC (2005). Theoretical study of the antioxidant activity of vitamin $\mathrm{E}$ : reactions of $\alpha$-tocopherol with the hydroperoxy radical. J. Chem. Theory Comput., 1, 337-344.

Oteiza PI, Fraga CG, Mills DA, Taft DH (2018). Flavonoids and the gastrointestinal tract: Local and systemic effects. Mol. Asp. Med., 61, 41-49.

Rimbach G, De Pascual-Teresa S, Ewins BA, Matsugo S, Uchida Y, Minihane AM, Turner R, Vafeiadou K, Weinberg PD (2003). Antioxidant and free radical scavenging activity of isoflavone metabolites. Xenobiotica, 33, 913-925. 
Rüfer CE, Kulling SE (2006). Antioxidant activity of isoflavones and their major metabolites using different in vitro assays. J. Agric. Food. Chem. 54, 2926-2931.

Setchell KDR, Clerici C, Lephart ED, Cole SJ, Heenan C, Castellani D, Wolfe BE (2005). Sequol, a potent ligand for estrogen receptor $\beta$, is the exclusive enantiomeric form of the soy isoflavone metabolite produced by human intestinal bacterial flora. Am. J. Clin. Nutr., 81, 1072-1079.

Sun W, Saeys M (2008). First principles study of the reaction of formic and acetic acids with hydroxyl radicals. J. Phys. Chem. A, 112, 6918-6928.

Tejero I, Gonzalez-Garcia N, Gonzalez-Lafont A, Lluch JM (2007). Tunneling in green tea: Understanding the antioxidant activity of catechol-containing compounds. A variational transition-state theory study. J. Am. Chem. Soc., 129, 5846-5854.

Truhlar DG (1983). Current status of transition-state theory. J. Phys. Chem., 87, 2664-2682.

Usharani D, Lacy DC, Borovik AS, Shaik S (2013). Dichotomous hydrogen atom transfer vs proton-coupled electron transfer during activation of $\mathrm{X}-\mathrm{H}$ bonds $(\mathrm{X}=\mathrm{C}, \mathrm{N}, \mathrm{O})$ by nonheme iron-oxo complexes of variable basicity. J. Am. Chem. Soc., 135, 17090-17104.

Villuendas-Rey Y, Alvarez-Idaboy JR, Galano A (2015). Assessing the protective activity of a recently discovered phenolic compound against oxidative stress using computational chemistry. J. Chem. Inf. Model., 55, 2552-2561.

Vo QV, Nam PC, Thong NM, Trung NT, Phan C-TD, Mechler A (2019). Antioxidant motifs in flavonoids: O-H versus C-H bond dissociation. ACS Omega, 4, 8935-8942.

Watanabe S, Uehara M (2019). Health effects and safety of soy and isoflavones, in: The role of functional food security in global health. Singh RB, Watson RR, Takahashi T (Eds.). Elsevier Inc., Amstardam, Chapter 22, pp. 379-394.

Wiseman H, O'Reilly JD, Adlercreutz H, Mallet AI, Bowey EA, Rowland IR, Sanders TAB (2000). Isoflavone phytoestrogens consumed in soy decrease $F_{2}$-isoprostane concentrations and increase resistance of low-density lipoprotein to oxidation in humans. Am. J. Clin. Nutr., 72, 395-400.

Yuan J-P, Wang J-H, Liu X (2007). Metabolism of dietary soy isoflavones to equol by human intestinal microflora - implications for health. Mol. Nutr. Food Res., 51, 765-781.

Zhang H-Y, Ji H-F (2006). How vitamin E scavenges DPPH radicals in polar protic media. New J. Chem., 30, 503-504.

Zhang H-Y, Wang L-F (2002). Are allylic hydrogens in catechins more abstractable than catecholic hydrogens? J. Am. Oil Chem. Soc., 79, 943-944.

Zhao Y, Gonzalez-Garcia N, Truhlar DG (2005). Benchmark database of barrier heights for heavy atom transfer, nucleophilic substitution, association, and unimolecular reactions and its use to test theoretical methods. J. Phys. Chem. A., 109, 2012-2018.

Zhao Y, Truhlar DG (2008). The M06 suite of density functionals for main group thermochemistry, thermochemical kinetics, noncovalent interactions, excited states, and transition elements: two new functionals and systematic testing of four M06-class functionals and 12 other functionals. Theor. Chem. Acc., 120, 215-241. 